\title{
Die nationale Bedingtheit der Folgen der Europäischen Integration
}

\section{Article by an MPIfG researcher}

Susanne K. Schmidt: Die nationale Bedingtheit der Folgen der europäischen Integration. In: Zeitschrift für Internationale Beziehungen 10(1), 43-67 (2003). Nomos

Welche Folgen hat die europäische Integration für die Mitgliedsstaaten? Dieser Artikel zeigt, wie die Verflechtung mit der EU die institutionellen Handlungsoptionen nationaler Akteure verändert. Der Ausgangspunkt ist das deutsche politische System, das aufgrund vielfältiger Verhandlungszwänge zu Reformstau neigt. Policies können deshalb stabil bleiben, auch wenn sie nur noch von einer Minderheit relevanter Akteure unterstützt werden. Am Beispiel des Versicherungswesens und des Straßengüterverkehrs wird gezeigt, wie erst die Einbindung in das europäische Mehrebenensystem es erlaubte, Reformen durchzusetzen, für die ohne die EU-Mitgliedschaft auf nationaler Ebene der notwendige Konsens gefehlt hätte. Analysiert man in dieser Weise die Folgen der Integration nicht nur als einseitigen Druck auf die Mitgliedsstaaten, sondern bezieht die Reaktionen nationaler Akteure ein, wird deutlich, wie sehr die Auswirkungen der EU durch nationale Interessen und institutionelle Strukturen bestimmt sind. Die Einordnung in das europäische Mehrebenensystem, so die These, erzeugt nationale Interaktionseffekte bzw. Resonanz.

\section{Die Folgen der europäischen Integration für die Mitgliedsstaaten ${ }^{1}$}

Die Forschung zur Europäischen Union (EU) nimmt sich zunehmend der Folgen des Integrationsprozesses für die Mitgliedsstaaten an. ${ }^{2}$ Als gemeinsamer Fokus verschiedener Arbeiten hat sich in letzter Zeit die Frage nach der Passgenauigkeit zwischen Politiken oder institutionellen Strukturen auf nationaler und europäischer Ebene herausgestellt. ${ }^{3}$ Abhängig hiervon wird der Anpassungsdruck gesehen, der auf der nationalen Ebene lastet. Ohne Anpassungsdruck ergibt sich keine nationale Veränderung, lautet das Argument, so dass sich die nationalen Folgen der Integration über diesen Druck manifestieren.

Ein Nachteil dieses Fokus ist, dass von einem einseitigen Druck auf die Mitgliedsstaaten ausgegangen wird, der dem tatsächlich bestehenden wechselseitigen Ver-

1 Der Aufsatz beruht auf einem Vortrag auf der gemeinsamen Tagung der DVPW, ÖGPW und SVPW, »Der Wandel föderativer Strukturen«, Humboldt-Universität Berlin, 8. bis 9.Juni 2001. Ich danke den Teilnehmern der Diskussion, vor allem Sonja Puntscher-Riekmann sowie Roland Czada, Philipp Genschel, Simone Leiber, Susanne Lütz, Detlef Sack, Fritz Scharpf, Gerald Schneider und Oliver Treib, sowie drei anonymen GutachterInnen der ZIB für sehr hilfreiche Kommentare. Ebenfalls zu danken habe ich Roman Claren für Forschungsassistenz.

2 Vgl. dazu Héritier (1997); Schmidt (1997); Knill/Lenschow (1998); Knill/Lehmkuhl (1999); Börzel/Risse (2000).

3 Börzel/Risse (2000); Knill/Lehmkuhl (1999; Knill/Lenschow (1998); Schmidt (1999). 
hältnis wenig Rechnung trägt. Mitgliedsstaatliche Akteure sind nicht nur maßgeblich am Zustandekommen europäischer Regeln beteiligt, Akteure auf der nationalen Ebene nutzen auch den durch die EU-Mitgliedschaft veränderten institutionellen Rahmen als Opportunitätsstruktur, um Interessen durchzusetzen, die im rein nationalen Kontext gescheitert wären. Während die vorgenannten Arbeiten schwerpunktmäßig institutionelle Parallelen und Unterschiede auf nationaler und europäischer Ebene zur Erklärung der Folgen der Integration heranziehen, wird in diesem Artikel Akteursstrategien ein größerer Stellenwert eingeräumt. Dies entspricht einer Forderung, die in jüngster Zeit in der »Europäisierungsliteratur « verstärkt gestellt wird (Börzel/Risse 2000; Dimitrova/Steunenberg 2000: 207).

Die nationalen Folgen der Integration, die Europäisierung - so die These des Aufsatzes -, lassen sich nicht nur als Konsequenz eines auf den Mitgliedsstaaten lastenden Anpassungsdrucks verstehen. Vielmehr lassen sich die Folgen besser mit dem Begriff der »Resonanz « konzeptualisieren. Durch die europäische Integration werden nationale Entwicklungen angestoßen oder auch verlangt; die konkreten Auswirkungen hängen aber davon ab, wie mit diesen Anforderungen - vor dem Hintergrund nationaler Erfahrungen - umgegangen wird und wie sie interpretiert werden. Es kommt also auf die nationalen institutionellen Bedingungen und die vor diesem Hintergrund agierenden Akteure an. Analysiert werden die Folgen der Integration anhand des deutschen politischen Systems, das bekanntermaßen aufgrund seiner vielfältigen institutionellen Verflechtungen Akteure zu einer gewissen Immobilität zwingt. Der föderative Aufbau mit der starken Rolle des Bundesrats führt zu vertikaler und horizontaler Politikverflechtung und zu einem »extreme[n] Konsenszwang der deutschen Politik « (Scharpf 1989: 127). Hinzu kommt der sektorspezifische Neokorporatismus, der durch die politikfeldspezifische Selbststeuerung weitere Blockademöglichkeiten nach sich zieht. Deutsche Politik ist so von starken Verhandlungszwängen geprägt, was sich auch mit dem Konzept von Vetopunkten (oder -spielern) fassen lässt (Czada 2000: 16f). Hierdurch kommt es leicht zu »Reformblockaden«, verstanden als eine »strukturelle Unfähigkeit der deutschen Politik, ernsthaft gewollte Veränderungen auch politisch durchzusetzen« (Scharpf 1989: 125). In seinem bekannten Buch hat Katzenstein (1987) diesen Sachverhalt als Teil der Semisouveränität der Bundesrepublik gefasst und über verschiedene Politikfelder gezeigt, wie diesen Systemeigenschaften inkrementelle Reformen entsprechen.

Aus der Perspektive der analytischen Politik lässt sich dieser Inkrementalismus präzise fassen. Denkt man sich die Präferenzen der Akteure auf einer Linie, die verschiedene Liberalisierungsstufen repräsentiert, muss für eine Reform des Status quo ein Punkt gefunden werden, den alle relevanten Akteure präferieren bzw. dem gegenüber sie - verglichen mit dem Status quo - indifferent sind (Hinich/Munger 1997: 21-71). Ein politisches System mit vielen Vetopunkten neigt entsprechend zu sehr inkrementellen Reformen, geprägt von vielfältigen Kompromissen (Moe/Caldwell 1994: 174). Aus dieser Perspektive wird deutlich, dass es abhängig von den Präferenzen der verschiedenen Vetospieler zu Kompromissen oder zu Nicht-Einigung (»Reformstau «) $)^{4}$ kommen kann. Teilen alle Vetospieler die Präferenz für eine 
weitergehende Reform des Status quo, ist auch dies möglich; es ist jedoch sehr unwahrscheinlich, wie auch Katzensteins (1987) Analysen gezeigt haben.

Wie wirkt sich nun die Verflechtung mit der EU auf diesen Zusammenhang aus? Die Blockadetendenzen können durch die weitere Verflechtung mit der EU überwunden werden. Dabei handelt es sich bei meinen Beispielen nicht um einen einseitigen supranationalen Druck von der EU, der nationale Änderungen erzwingt. Vielmehr wird die Verflechtung mit der EU von nationalen Akteuren strategisch genutzt, um Reformen durchzusetzen, für die ohne die EU-Mitgliedschaft national der notwendige Konsens fehlte. ${ }^{5}$ Durch die Verflechtung mit der zusätzlichen europäischen Ebene werden nationale Entwicklungen angestoßen, die helfen, einen gewachsenen Reformstau zu überwinden. ${ }^{6}$ Die Mitgliedschaft erzeugt auf der nationalen Ebene Resonanz.

Diese Folgen der europäischen Integration werden anhand zweier hochregulierter Politiken diskutiert: dem Versicherungswesen und dem Straßengüterverkehr. Ausgewählt wurden zwei Politikfelder mit einer ähnlichen analytischen Problematik, die sich jedoch von ihren Sektorstrukturen stark unterscheiden (bezogen auf die Unternehmensgröße, Komplexität der Dienstleistungen, Langfristigkeit der Verträge). Damit kann zwar nicht dem »most similar« bzw. »most different case design « (Przeworski/Teune 1985) entsprochen werden, da passende Fälle in diesem Bereich nicht vorhanden sind. Allerdings ist die empirische Basis verglichen mit den bestehenden Studien, die sich fast immer nur auf ein Politikfeld beziehen (meist die Umweltpolitik, vgl. Börzel 2002; Knill/Lenschow 1998) deutlich verbreitert. In beiden Politikfeldern gelang es der Bundesregierung nicht, die bestehende Regulierung kontinuierlich neuen Anforderungen anzupassen. Die Regulierung des Versicherungswesens hatte sich Anfang des 20. Jahrhunderts ausgebildet und blieb seitdem weitgehend intakt. Ähnliches lässt sich für den Straßengüterverkehr mit einer entsprechend kürzeren Tradition behaupten. Obwohl in den Siebziger- und Achtzigerjahren die Reformnotwendigkeit besonders des Straßengüterverkehrs immer offensichtlicher wurde, fehlte der notwendige Konsens, um den pathologischen Auswirkungen der Regulierung zu begegnen. Die Reform gelang erst angesichts der Vorgaben der EU. Überdies wurden gleichzeitig weitergehende Veränderungen umgesetzt, so dass es zu einem erheblichen Umbau der Sektorregulierung kam.

4 Luthardt (1999: 12) erinnert daran, dass 1997 das Wort »Reformstau« von der Gesellschaft für deutsche Sprache zum »Wort des Jahres « gewählt wurde.

5 In diesem Punkt unterscheide ich mich von der Analyse von Wachendorfer-Schmidt (1999), die auch auf die Möglichkeit eingeht, deutsche Reformblockaden durch die EU zu überwinden, hier aber weniger den aktiven Part der nationalen Ebene betont (Wachendorfer-Schmidt 1999: 16, 27).

6 Angesichts des bekannten Aufsatzes zur Politikverflechtungsfalle in der EG (Scharpf 1985) drängt sich die Frage auf, wie es trotz institutioneller Ähnlichkeiten zu einer handlungsfähigeren europäischen Ebene kommen kann. Die Erklärung liegt vor allem in der negativen Integration und der Bedeutung von Richterrecht, wodurch Liberalisierungsentscheidungen entweder nicht der Zustimmung des Ministerrats bedürfen oder aber dieser unter dem »Schatten« des Europäischen Gerichtshofs handelt (vgl. Schmidt 2000). Ich führe diesen Punkt unten weiter aus. 
Damit handelt es sich nicht nur um die Folgen eines einseitigen supranationalen Drucks, wodurch eine auf die institutionelle Passgenauigkeit ausgerichtete Konzeptualisierung zu kurz greift.

Die Stoßrichtung dieses Artikels ist eine doppelte: Zum einen geht es um die Folgen der Integration für die Bundesrepublik. Die zusätzliche Verflechtung mit der EU kann den Wandel fördern, weil bisherige, institutionell gestützte Vetokoalitionen aufgebrochen werden können. Die Verknüpfung des nationalen mit dem europäischen politischen System verändert die Handlungsoptionen nationaler politischer Akteure, da manche Akteure bestehende nationale institutionelle Restriktionen durch das europäische Mehrebenensystem umgehen und so die Durchsetzungsmöglichkeiten ihrer Interessen steigern können. Zum anderen soll auf dieser Basis ein Beitrag zur Europäisierungsliteratur geleistet werden, welche die durch die Integration auf nationaler Ebene angestoßenen Entwicklungen, kurz die Resonanz, bisher ungenügend thematisiert.

Im Folgenden geht es zunächst um das ursprüngliche Regulierungsmodell für den Straßengüterverkehr und das Versicherungswesen in Deutschland. Danach wird die Politik der EU geschildert und die Reaktion in Deutschland analysiert. Das Ineinandergreifen des europäischen und des nationalen Politikprozesses verdeutlicht, dass es sich nicht um einen einseitigen Anpassungsdruck auf das deutsche System handelt, sondern dass die europäische Verflechtung von manchen Akteuren strategisch genutzt wird, um ihre Verhandlungsposition zu verbessern und bestehende Vetokoalitionen zu umgehen. Abschließend wird diskutiert, inwieweit die Ergebnisse über die hier vorgestellten zwei Fälle hinaus verallgemeinerbar sind und welche Schlüsse daraus für die Europäisierungsliteratur zu ziehen sind.

\section{Die ursprüngliche deutsche Regulierung von Versicherungen und Straßengüterverkehr}

Versicherungen und Straßengüterverkehr gehören in der Bundesrepublik zu den Materien, die der Zustimmung des Bundesrates bedürfen. Die ursprüngliche Regulierung in beiden Bereichen war recht ähnlich. Sowohl Versicherungen als auch der Straßengüterverkehr waren in Deutschland traditionell stark reguliert. Die Regulierung erfolgte in beiden Bereichen in enger Abstimmung des zuständigen Bundesamtes mit den Verbänden und trug so mesokorporatistische Züge. Reguliert waren die Marktzulassung, die Tarife, der laufende Betrieb und bei Versicherungen auch die Produkte. Hieraus folgten zum einen ein Markt mit geringer Wettbewerbsintensität und zum anderen eine weitgehende Interessenkongruenz zwischen Regulierungsbehörde und Regulierten, was das Regulierungsmodell stabilisierte. 


\subsection{Die Regulierung des Versicherungswesens}

Die Versicherungsregulierung in der Bundesrepublik ist vor allem im Versicherungsaufsichtsgesetz (VAG) von 1901 geregelt. Das VAG ist zustimmungspflichtig ebenso wie das Gesetz über die Einrichtung eines Bundesaufsichtsamts für das Versicherungswesen, ${ }^{7}$ mit dem 1951 das BAV gegründet wurde, wofür man sich weitgehend am Vorläufer des Reichsaufsichtsamts für das Versicherungswesen orientierte. Das BAV unterstand zunächst dem Bundeswirtschaftsminister und wechselte Ende 1972 in die Zuständigkeit des Bundesfinanzministers.

Die deutsche Regulierung des Versicherungswesens war äußerst umfassend und beinhaltete die Genehmigung der allgemeinen Versicherungsbedingungen und neuer Versicherungsprodukte ebenso wie die Kontrolle der Kalkulation von Prämien und Rückstellungen in der Lebens-, Kranken- und Pflichtversicherung (Krakowski 1988). Grundlegend für die sehr weitgehende materielle Staatsaufsicht war eine Generalklausel im VAG ( $\$ 81$, Abs. 2, 1), die der Aufsichtsbehörde die Missstandsaufsicht zusprach (Geiger 1992: 85). Die Regulierung hatte zur Folge, dass der deutsche Versicherungsmarkt weitgehend vereinheitlicht war. Ein Preisvergleich war jedoch nur möglich, wenn neben den Prämien die Überschussbeteiligungen und Beitragsrückerstattungen einbezogen wurden. Damit war für den Verbraucher trotz Regulierung wenig Markttransparenz gegeben. Die Regulierung beruhte auf einer engen Zusammenarbeit des BAV mit den Versicherungsverbänden, dem Gesamtverband der Deutschen Versicherungswirtschaft (GDV) sowie den Fachverbänden für die einzelnen Versicherungssparten. Die Prämienanpassungen und die Versicherungsbedingungen wurden gemeinsam erarbeitet (Krakowski 1988: 459). Diese weitgehende Einbindung der Verbände war möglich, weil für Versicherungen eine Bereichsausnahme vom Gesetz gegen Wettbewerbsbeschränkungen (GWB) galt. Das Bundeskartellamt war nur im Rahmen einer Missbrauchsaufsicht in das Versicherungswesen involviert, die im Einvernehmen mit dem BAV durchzuführen war (Hollenders 1985).

Die Regulierung war insofern erfolgreich, als es kaum zu Insolvenzen in der Versicherungsbranche kam. Allerdings waren die Prämien im internationalen Vergleich sehr hoch, und Produktinnovationen blieben aus. Wegen der verbreiteten Praxis von zehnjährigen Verträgen ohne Kündigungsrecht, für die die Versicherer in den Achtzigerjahren außerdem vermehrt in den Genuss von automatischen Prämienanpassungsklauseln kamen, gab es starke Kritik von Seiten der Verbraucherverbände. Benachteiligt fühlten sich somit von der herrschenden Regulierung jene, deren Schutz in der Begründung der Versicherungsaufsicht eine besondere Rolle spielte. $\mathrm{Zu}$ den Reformbefürwortern zählte auch die Wirtschaft: Zwar profitierte sie von recht günstigen Prämien, da die Industrieversicherung in der Regel von der Massenversicherung quersubventioniert wurde (Dreher 1996: 501); aber durch die umfassende Regulierung waren die Bedingungswerke starr und entsprachen nicht den Wünschen der Industrie nach einem flexibleren Versicherungsschutz. Dieser

7 Das Versicherungsvertragsgesetz ist dagegen nicht zustimmungspflichtig. 
Zustand, der eindeutige Züge von regulatory capture (Stigler 1971; Peltzman 1989) trug, blieb nicht ohne Kritik. So gab es Mitte der Achtzigerjahre einen öffentlich ausgetragenen Streit zwischen dem Bundeskartellamt und dem BAV über die automatischen Prämienanpassungsklauseln, in dem das BAV dem Bundeskartellamt schließlich eine größere Mitsprache zugestand. ${ }^{8}$ Auch im Rahmen verschiedener Kartellnovellen wurde diskutiert, ob Versicherungen aus der Ausnahme des Paragraphen 102 herausfallen sollten, so bei der 2. Kartellnovelle 1970 und bei der 4 . Kartellnovelle 1980 (Hollenders 1985: 47f). Außerdem forderte die Monopolkommission in ihrem Hauptgutachten 1986/1987 weitgehende Änderungen in der Versicherungsregulierung (Monopolkommission 1988: 30-33, 245f). 1989 wurde der Paragraph 102 mit der 5. Kartellnovelle aber nur geringfügig geändert (Starke 1988: 1230). Schließlich unterbreitete die Deregulierungskommission in ihrem Bericht detaillierte Vorschläge zu einer Liberalisierung des Versicherungswesens (Deregulierungskommission 1991: 28-34). Ihre Umsetzung wurde jedoch nicht weiter diskutiert, weil zu diesem Zeitpunkt die Arbeiten der EG-Kommission schon weit gediehen waren.

Wie lässt sich die Kontinuität der Versicherungsregulierung in Deutschland erklären? Zunächst erlaubte die Marktabschottung der Politik, die Anlage der Mittel weitgehend auf den heimischen Markt zu begrenzen und Vorgaben für die Investition in Staatstitel zu machen. Das war nach dem Zweiten Weltkrieg ebenso wichtig wie zuvor, was es erleichterte, das aus der Vorkriegszeit stammende Regulierungsmodell mit dem neuen ökonomischen Liberalismus zu verbinden (Everson 1996: 212). Des Weiteren ist die Ressortzuständigkeit zu nennen (vgl. Mayntz/Scharpf 1975: 42, 69, 74): Nach dem Wechsel in das Bundesfinanzministerium (BMF) gab es innerhalb des Ministeriums kein prinzipielles Interesse an einer Liberalisierung, während man dies bei einer weiteren Zuständigkeit des Bundeswirtschaftsministeriums (BMWi) der dortigen Grundsatzabteilung hätte unterstellen können. Aus dem federführenden Ministerium kamen so keine nennenswerten Reforminitiativen. Außerdem muss auf die Parallelität der Interessen des Gewerbes mit denen der Politik verwiesen werden: Als sehr finanzstarke Branche fiel es der Versicherungswirtschaft anscheinend leicht, politisch Gehör zu finden. Mitte der Achtzigerjahre wurde ein Spendenskandal aufgedeckt, bei dem mehr als hundert Politiker großzügig gefördert worden waren. ${ }^{9}$ Auch haben die Unternehmen die mit der Aufsicht verbundenen Restriktionen nie bekämpft. Die Verbände hatten eine starke Stellung, da das BAV vielfältig auf ihr Wissen zurückgriff und Neuerungen gemeinsam mit ihnen erarbeitete. Aufgrund ihrer hervorgehobenen Rolle war der Organisationsgrad hoch: »Kaum ein Bedingungswerk und kaum ein Tarifbuch kommt ohne Verbandshilfe zustande. Es kann daher nicht verwundern, dass selbst kleinste ausländische Niederlassungen sich umgehend den entsprechenden Verbänden anschließen«

8 Siehe »Kartellamt will Versicherte schützen«, Süddeutsche Zeitung, 28.1.1985; »Mehr Wettbewerb über neue Produkte gefordert«, Handelsblatt, 7.3.1986; »Versicherungs-Aufsicht will Kündigungsrecht verbessern«, Süddeutsche Zeitung, 15.5.1986.

9 »Versicherungen: Das ganze System ist schief«, Der Spiegel, 26.8.1985. 
(Eggerstedt 1986: 20). Schließlich sind die Bundesländer zu nennen, die aufgrund der Zustimmungspflichtigkeit des VAG einbezogen waren. Hier lässt sich naturgemäß nicht dieselbe einheitliche Linie wie bei den Verbänden ausmachen. Generell vertreten Bundesländer, in denen wichtige Versicherungsstandorte liegen, die entsprechenden Interessen (int 5). ${ }^{10}$ Darüber hinaus gab es immer wieder Initiativen aus den Bundesländern, die zeigen, dass die Länder bei der Reform der Versicherungsaufsicht durchaus eigene Ziele verfolgten. ${ }^{11}$ Selbst in der Wissenschaft gab es kaum Kritik an der Versicherungsregulierung: ${ }^{12}$

»Es gibt keinen bekannten Autor, der nicht zugleich Mitglied der Aufsichtsbehörde oder Funktionsträger in einem Versicherungsunternehmen gewesen wäre oder der Branche in irgendeiner Weise verbunden ist. Auch die meisten wissenschaftlichen Einrichtungen werden von der Branche getragen. [...] So ist es nicht verwunderlich, dass im Schrifttum eine konservativ-zustimmende Richtung vorherrscht« (Gärtner 1984: 524).

Insgesamt war die Versicherungsregulierung in Deutschland so von großer Kontinuität und Stabilität gekennzeichnet. Akteure, die die Ausrichtung kritisierten, wie die Verbraucherschutzverbände und das Bundeskartellamt, konnten sich mit Änderungsvorschlägen kaum gegen das Bundesaufsichtsamt und die Versicherer durchsetzen.

\subsection{Die Regulierung des Straßengüterverkehrs}

Die Regulierung des Straßengüterverkehrs weist eine ähnliche Kontinuität wie die des Versicherungswesens auf. Das zustimmungspflichtige Güterkraftverkehrsgesetz (GüKG) von 1952 schloss bestehende Regelungslücken der Nachkriegsregulierung und gründete die Bundesanstalt für den Güterfernverkehr (BAG; Feick et al. 1982: 190f, 200). Die BAG verdankte sich der Initiative des Gewerbes, das nach Schwierigkeiten mit einer vorherigen freiwilligen Kontrolle an den Gesetzgeber herangetreten war (Laaser 1991: 164).

Mit wenigen Änderungen blieb die durch das GüKG geschaffene bzw. fortgeschriebene Marktregulierung bis in die Neunzigerjahre intakt. Für diese Kontinuität war vor allem die fest gefügte Akteurskonstellation verantwortlich, die diese Marktordnung unterstützte (Hamm 1992: 226). Dazu gehörte zunächst das Bundesver-

10 Bezieht sich auf Interview Nr. 5 aus 22 qualitativen Interviews, die zwischen Januar 2001 und März 2002 vorwiegend mit Beamten in den zuständigen Ministerien bzw. der Europäischen Kommission und Vertretern von Sektorinteressen auf nationaler und europäischer Ebene geführt wurden. Im folgenden als »int $1 \ll$ bis »int 22 « gekennzeichnet.

11 Beispielsweise startete die niedersächsische Landesregierung 1986 eine Initiative zur Reform der Anlagevorschriften; und die Einigung auf die Umsetzung der 3. Richtlinien erfolgte 1994 erst im Vermittlungsausschuss (»Die Entscheidungen des Kartellamts sind dem Verbraucher nicht immer dienlich«, Handelsblatt, 6.11.1986; »Länder wollen Fesseln lockern«, Frankfurter Rundschau, 8.6.1994; »Gesetze für höhere deutsche Beschäftigung «, Neue Züricher Zeitung, 29.6.1994).

12 Dies änderte sich etwas ab 1983, nachdem eine Arbeit Finsingers starke Kritik an der Versicherungsregulierung übte (Bujard 1991: 192). 
kehrsministerium als einflussreichster Entscheidungsträger, das sehr enge Klientelbeziehungen unterhielt (Garlichs/Müller 1977: 344f). Als bundesunmittelbare Anstalt des öffentlichen Rechts war die Bundesanstalt für Güterfernverkehr für die Einhaltung der Güterverkehrsregulierung zuständig. Neben der Überwachungsfunktion nahm sie Ordnungsaufgaben wahr und übte beratende und statistische Tätigkeiten aus (Müller/Vogelsang 1979: 235). ${ }^{13}$ Die BAG war explizit als »Organisation der Selbstverwaltung des Gewerbes « geplant worden. ${ }^{14}$ Entsprechend arbeitete sie eng mit den verschiedenen Verbänden des Transportgewerbes zusammen. Die Regulierung bezog sich auf den Marktzugang und den Preis. Als Begründung diente die Lehre von den $»$ Besonderheiten des Verkehrs $«{ }^{15}$ und der Schutz der Bahn. Die Regulierung unterteilte den Markt in den Fernverkehr (außerhalb eines 50-KilometerRadius um den Lkw-Standort), den Nahverkehr (innerhalb dieses Radius), Umzugsverkehr und Werkverkehr. Der Fernverkehr war am stärksten reguliert. Die Konzessionen waren kontingentiert und zudem wurden Margentarife und Vertragskonditionen vorgeschrieben. Für den Nah- und Umzugsverkehr gab es dagegen nur Margentarife und subjektive Marktzugangsbeschränkungen. Der Werkverkehr war kaum reguliert (Monopolkommission 1990: 693). Die Tarife wurden von Tarifkommissionen festgesetzt, deren Vertreter vom Bundesverkehrsminister bestimmt wurden, indem faktisch die Vorschläge der entsendenden Verbände einfach bestätigt wurden. Bemerkenswert war, dass in der Kommission für den Fernverkehr nur Vertreter des Transportgewerbes saßen. Ein gesonderter Verladerauschuss hatte eine rein beratende Rolle. Allerdings mussten die Tarife vom Bundesverkehrs- und vom Bundeswirtschaftsminister genehmigt werden, so dass die Verladerinteressen stärker berücksichtigt wurden, als es ihrer direkten Beteiligung entsprach (Feick et al. 1982: 232f; Lehmkuhl 1998: 152; Hamm 1984: 473). Die Aufsicht über die korrekte Anwendung der Tarife war vom BAG an die Straßenverkehrsgenossenschaften delegiert worden, die den wirtschaftlichen Arm der Transportverbände bildeten. Die daraus anfallenden Einnahmen waren eine wichtige Finanzquelle für die Verbände (Lehmkuhl 1998: 150f). Mit dieser Betrauung des Gewerbes versuchte man, sich dessen Eigeninteresse an einer möglichst korrekten Implementation zunutze zu machen: Das Gewerbe profitierte von möglichst hohen regulierten Tarifen und war daran interessiert, durch eine genaue Umsetzung die Notwendigkeit weiterer Regulierung auszuräumen (Feick et al. 1982: 236; Wacker-Theodorakopoulos 1988: 315).

Auch im Straßengüterverkehr findet sich also eine capture-Situation. Benachteiligt wurden von diesem Regulierungssystem vor allem die Verlader. Angesichts der fest gefügten »Proregulierungsfraktion « von Transportgewerbe, BAG und Bundesverkehrsministerium mussten sie sich weitgehend darauf bescheiden, Angriffe auf den Werkverkehr abzuwehren und etwas mäßigenden Einfluss auf die Tarife auszu-

13 Ansonsten oblag der Vollzug des Güterkraftverkehrsgesetzes den Landesbehörden.

14 Deutscher Bundestag 1949, Begründung zum Entwurf des GüKG, zitiert aus Laaser (1991: 154).

15 Dazu gehört z. B., dass Verkehrsleistungen nicht stapelbar sind, saisonalen Schwankungen unterliegen und unpaarige Verkehrsströme zu einem Rückfrachtproblem führen (Deregulierungskommission 1991: 39). 
üben (Willeke 1997: 55). Einen Fürsprecher fanden die Verlader im Bundeswirtschaftsministerium. Weiterhin müssen zur Liberalisierungsfraktion auch viele Vertreter der Verkehrswissenschaft gezählt werden (Teutsch 2001: 138). Seit den Siebziger- und Achtzigerjahren setzte sich eine Mehrheit der Disziplin für eine Deregulierung ein (Willeke 1997: 56, 63). Und wie wir sehen werden, hatten akademische Vertreter, insbesondere Europarechtler, mit ihrer Argumentation Einfluss auf die vorzeitige Lockerung des deutschen Regulierungsmodells.

Während die Bahn trotz der Regulierung zunehmend defizitär wurde, entstanden eine Reihe von Fehlentwicklungen durch die Regulierung (Aberle 1987: 164). So lagen die Preise etwa 20\% über den vermuteten Marktpreisen. Der Werkverkehr stieg überproportional an und führte zu häufigen Leerfahrten. Auch der graue Konzessionshandel zeigte, dass die künstliche Angebotsverknappung erhebliche Gewinnmargen erlaubte - für eine Konzession wurden bis zu 300.000 D-Mark gezahlt (Monopolkommission 1990). Selbst ausländische Seehäfen profitierten zu Lasten deutscher Häfen, da sich ein Auslandstransport tariflich oft lohnte (WackerTheodorakopoulos 1988: 335).

Angesichts dieser Fehlentwicklungen wurde die Güterkraftverkehrsregulierung zunehmend kritisiert. In den Achtzigerjahren entstanden eine Reihe von Studien, die eine Deregulierung des Verkehrswesens unter Effizienzerwägungen forderten (Teutsch 2001: 138). Auch die Monopolkommission machte in ihrem Hauptgutachten 1988/1989 Vorschläge zur Deregulierung und hob die eingetretenen Fehlentwicklungen hervor (Monopolkommission 1990). Im Bericht der Deregulierungskommission folgte eine weitere Analyse mit der Forderung nach Reformen (Deregulierungskommission 1991). So wurde die bisherige Begründung der weitgehenden Regulierung mehr und mehr kritisiert.

Dennoch gab es keine politischen Reforminitiativen. Das Bundeswirtschaftsministerium, die Verlader und die Wirtschaft insgesamt, die für eine Reform eintraten und die Kosten der Fehlentwicklungen zu tragen hatten, konnten sich gegen die fest gefügte Koalition der am Status quo interessierten Akteure alleine nicht durchsetzen. Zur Erklärung muss man auch hier zunächst auf die Ressortautonomie und die Struktur der Bundesministerien verweisen (vgl. Mayntz/Scharpf 1975: 42, 69, 74). Das federführende Bundesverkehrsministerium hielt aufgrund der engen Beziehungen zum Transportgewerbe und der BAG an der Regulierung fest. Dagegen konnte das reforminteressierte BMWi nur im Rahmen allgemeiner Initiativen wie der Arbeit der Deregulierungskommission eingreifen. Zudem war auch der zuständige Bundestagsausschuss von den Gewerbeinteressen dominiert (Müller-Rommel 1988: 309).

Die Position der Länder lässt sich nicht eindeutig der Pro- oder der Kontra-Liberalisierungsfraktion zuordnen. Zum einen gab es bereits 1982 einen Vorstoß aus Niedersachsen gegen die Tarifbindung, auch forderten die nördlichen Bundesländer Ausnahmen hiervon, um ihre Häfen zu fördern. ${ }^{16}$ Zum anderen gab es in den Län-

$16 »$ Angriff auf Tarifbindung«, Handelsblatt, 29.7.1982; »Seehäfen: Voll da«, Der Spiegel, 24.6.1985. 
dern wiederholt Widerstand gegen eine Lockerung der Marktordnung, und der Bundesrat setzte sich 1997 zusammen mit dem Gewerbe erfolgreich für eine niedrigere Geltungsgrenze (ab 3,5 statt ab 6 Tonnen) des reformierten GüKG ein. ${ }^{17}$ Dahinter standen sowohl Interessen an der Mittelstandsförderung als auch Befürchtungen, dass eine Aufhebung der Marktordnung zu einer Benachteiligung der Fläche führen würde. Insgesamt ergab sich so die Situation, dass eine Regulierung beibehalten wurde, die nur etwa 5\% des Lastwagenbestands erfasste (Deregulierungskommission 1991: 46) und dabei erhebliche Folgekosten hatte.

\section{Die europäische Politik}

Bis zur Binnenmarktinitiative und dem Übergang zu qualifizierten Mehrheitsentscheidungen im Ministerrat machte die gemeinsame Politik in beiden Bereichen nur wenig Fortschritte. Eine vorherige Harmonisierung wurde vor allem im Verkehrsbereich durch ein Junktim vom Ministerrat explizit zur Voraussetzung für eine Liberalisierung gemacht (Schmitt 1993: 305f).

Im Versicherungswesen einigte man sich 1964 auf eine erste Richtlinie, die die national kaum regulierte Rückversicherung betraf. In den Siebzigerjahren verwirklichte man durch zwei Richtlinien die Niederlassungsfreiheit für die Schadens- und die Lebensversicherung (Badenhoop 1988: 103). Damit konnten Agenturen oder Zweigniederlassungen in anderen Mitgliedsstaaten unter denselben Zulassungs- und Tätigkeitsbedingungen errichtet werden, die für Inländer vorgeschrieben waren.

Für den Straßengüterverkehr einigte man sich in verschiedenen Verordnungen in den Sechziger- und Siebzigerjahren auf ein gemeinsames Tarifsystem und ein Gemeinschaftskontingent von Genehmigungen, die zu Transporten zwischen beliebigen Orten in der Gemeinschaft berechtigten (Button 1984: 42f, 77-79). Daneben existierten bilaterale Kontingente zwischen den Mitgliedsstaaten. Die Fiskalharmonisierung sowie die soziale ${ }^{18}$ und technische ${ }^{19}$ Harmonisierung standen außerdem auf der Tagesordnung, es wurden aber keine großen Fortschritte erzielt. Erst ab Mitte der Achtzigerjahre gelang es, die Dienstleistungsfreiheit im Straßengüterverkehr und im Versicherungswesen schrittweise durchzusetzen.

\subsection{Der Binnenmarkt für Versicherungen}

Neben dem Binnenmarktprogramm und dem Übergang zu qualifizierten Mehrheitsentscheidungen im Ministerrat war ein Urteil des Europäischen Gerichtshofes

17 Dr. Herbert Huber, MdL: »Schiene oder Straße: Entscheidung der Vernunft. Klarer ordnungspolitischer Rahmen«, Bayern-Kurier, 27.4.1985; »Güterkraftverkehr, Erfolg für Fuhrgewerbe: Korrektur gefordert«, Handelsblatt, 29.9.1997.

18 Diese umfasst Lenk- und Ruhezeiten sowie, für die Kontrolle, Fahrtenschreiber.

19 Maße und Gewichte schwerer Kraftfahrzeuge. 
(EuGH) für den Fortschritt des Versicherungsbinnenmarkts wichtig, das Ende 1986 erging. Auf das Urteil muss hier nur insofern eingegangen werden, als der EuGH zwar den Mitgliedsstaaten für den Massenversicherungsmarkt ein besonderes Schutz- und Harmonisierungsinteresse zubilligte, jedoch für den Bereich der Industrieversicherungen die Realisierung der Dienstleistungsfreiheit forderte (Geiger 1992: 240-282). Dieses Urteil erleichterte im Ministerrat die Annahme der seit 1975 vorliegenden Großschadenrichtlinie, die die Dienstleistungsfreiheit für Industrieversicherungen betraf. Eine vergleichbare Richtlinie für die Lebensversicherung (vor allem für Gruppenversicherungen) folgte. Darüber hinaus plante die Kommission mit zwei weiteren Richtlinien auch die Dienstleistungsfreiheit für den Massenversicherungsmarkt. Auf der Grundlage von harmonisierten Vorschriften für die Höhe des Eigenkapitals, der Vermögensanlage und der technischen Rückstellungen sollte für Versicherungen - ähnlich wie bereits für Banken - die Heimatlandaufsicht zuständig sein. Selbst auf diese so genannten dritten Richtlinien einigte sich der Ministerrat, so dass seit Mitte 1994 die Niederlassungs- und Dienstleistungsfreiheit auf Versicherungsmärkten verwirklicht ist. Auch das Bundesfinanzministerium stimmte zu, was sicherlich mit ausschlaggebend war für die sehr schnelle Verabschiedung, für die vom EuGH kein Druck ausging. Erklären muss man den deutschen Positionswechsel zunächst mit der dominanten neoliberalen Ideologie der Zeit (Knudsen 2001: 182) und dann mit der wachsenden Internationalisierung, die den »Finanzplatz Deutschland« zu einem wichtigen Thema werden ließ. Obwohl sich diese Diskussion vor allem auf die Banken und Börsen bezog, wurde die Versicherungsregulierung in diesem Kontext mit angesprochen. Zudem konnten aufgrund der von kleinen und mittleren Unternehmen geprägten Unternehmensstruktur in Deutschland verhältnismäßig viele Unternehmen nicht von der Liberalisierung der Großschadenrichtlinie profitieren, so dass von Seiten der Wirtschaft Interesse an einer weitergehenden Liberalisierung bestand. Schließlich war die deutsche Einigung soeben erfolgt, was zum Druck anderer Mitgliedsstaaten (vor allem Großbritanniens) führte, diesen neuen Markt nicht abzuschotten (int 4, int 5). ${ }^{20}$

\subsection{Der Binnenmarkt für Straßengüterverkehr}

Stärker als beim Versicherungswesen war beim Straßengüterverkehr ein Urteil des EuGH dafür ausschlaggebend, dass die Richtlinien zur Realisierung des Binnenmarkts verabschiedet werden konnten. Da es im EG-Vertrag ein gesondertes Kapitel zum Verkehr gibt, der Ministerrat aufgrund des Junktims von Harmonisierung und Liberalisierung sowie der unterschiedlichen Interessenlage jedoch den gemeinsamen Markt für Verkehr nicht verwirklichte, trat das Europäische Parlament mit

20 Vgl. die Financial Times »Anglo-German boost for single insurance market« vom 31.3.1990 zum Treffen der zuständigen Minister Ridley und Waigel, bei dem man sich auf den Versicherungsbinnenmarkt und die Grundsätze von gegenseitiger Anerkennung und Minimalharmonisierung verständigte. 
einer Untätigkeitsklage vor den EuGH. In seinem Untätigkeitsurteil betonte der EuGH die Verpflichtung des Ministerrats, die Dienstleistungsfreiheit für Verkehr innerhalb eines »angemessenen Zeitraums « zu realisieren (Erdmenger 1985: 378). Damit wurde das Junktim mit der Harmonisierung durchbrochen. ${ }^{21}$

Der Ministerrat einigte sich daraufhin, den grenzüberschreitenden Verkehr durch eine Aufhebung der Kontingente ab 1.1.1993 vollständig zu liberalisieren (Verordnung 1841/88). Gleichzeitig wurde eine Marktzugangsregelung eingeführt, die ausschließlich an subjektiven (in der Person des Bewerbers liegenden) Kriterien ansetzte (Basedow/Dolfen 1998: 72). Neben der Liberalisierung des grenzüberschreitenden Verkehrs musste aber auch die Dienstleistungsfreiheit innerhalb der Mitgliedsstaaten realisiert werden, was sich schwieriger gestaltete. Die Einigung auf die so genannte Kabotage war problematisch, weil sich damit Wettbewerbsverzerrungen zwischen den Mitgliedsstaaten nicht nur im grenzüberschreitenden, sondern auch im Inlandsverkehr auswirken würden (Wissenschaftlicher Beirat 1990: 6). Vor allem die Bundesregierung versuchte, sich dem zu widersetzen und durch gleichzeitige Harmonisierung die Bedingungen in den Mitgliedsstaaten anzugleichen. Angesichts des andauernden Konflikts um die Einführung der Kabotage drohten sowohl die Europäische Kommission als auch die Niederlande damit, den Ministerrat erneut vor dem EuGH zu verklagen (Wissenschaftlicher Beirat 1990: 8). Vor diesem Hintergrund gelang Ende 1989 die Einigung auf eine vorläufige Kabotageregelung (gültig bis Ende 1992), der - nach erneuten Drohungen der Kommission - Mitte 1993 ein Kompromisspaket folgte, das neben der endgültigen Kabotagefreigabe (ab Juli 1998/VO 3118/93) die gleichzeitige Verabschiedung einer Richtlinie über die einheitliche Besteuerung und die Erhebung von Straßenbenutzungsgebühren beinhaltete (Gronemeyer 1994: 271).

\section{Die Reaktion in Deutschland}

Mit den Richtlinien für den Binnenmarkt für das Versicherungswesen und den StraBengüterverkehr wurde die bisherige Regulierung in der Bundesrepublik radikal in Frage gestellt. Wie gezeigt, gab es zuvor zwar an beiden Regulierungssystemen Kritik von der Nachfrageseite (den Versicherungskunden bzw. den Verladern), dem Bundeswirtschaftsministerium, dem Bundeskartellamt, der Monopol- und der Deregulierungskommission, jedoch blockierte die Interessenkongruenz zwischen der Regulierungsbehörde und den Regulierten die Reformen. Solange das federführende Ressort an der Regulierung festhielt, blieben Reforminitiativen aus. Dieser erste Vetopunkt blieb beim Straßengüterverkehr die ganz Zeit intakt. Aber auch ein schließlich reformwilliges Ministerium wie das Bundesfinanzministerium sah sich weiteren Vetospielern gegenüber. Da sich die Versicherungswirtschaft gegen die Liberalisierung stellte, musste mit der Mobilisierung z. B. der Länder im Bundesrat gerechnet werden. Der Kontext der europäischen Politik änderte diese Situation. Für

21 Vgl. dazu EuGH 22.5.1985 - RS 13/83 (Parlament ./. Rat), Slg. 1985, 1513. 
die Liberalisierungsbefürworter bot sich nun die Gelegenheit, ihre Interessen voranzutreiben. Sei es, dass das Ministerium über die Zustimmung in Brüssel nationale Implementationszwänge herbeiführen konnte, oder dass Akteure das europäische Recht nutzten, um einen bestimmten Veränderungsdruck auf nationaler Ebene zu konstruieren; oder aber, dass der nun mögliche Wettbewerb mit weniger regulierten Unternehmen anderer Mitgliedsstaaten als Argument für eine Regulierungsänderung diente.

Im Versicherungswesen vollzog das Bundesfinanzministerium bereits bei der Umsetzung der Großschadenrichtlinie einen radikalen Schwenk. Die Richtlinie hatte die Liberalisierung in zwei Stufen vorgesehen; in der Bundesrepublik liberalisierte man den Markt gegen den Widerstand der Versicherungswirtschaft in einem Schritt (Finanzausschuss 1990: 26). Prinzipiell hatte die Versicherungswirtschaft die notwendige Liberalisierung des Industrieversicherungsmarkts im Zuge der Verwirklichung des Binnenmarkts eingesehen. Für den Massenmarkt hielt man aber ebenso wie das BAV an den hergebrachten Regulierungsprinzipien fest. Wie wir gesehen haben, verfolgte die Bundesregierung aber auch hier in Brüssel eine andere Politik und wehrte sich nicht mehr gegen eine Liberalisierung der Privatversicherungen, obwohl man, wie die Versicherungswirtschaft hervorhob, mit der bisherigen Linie einer Verteidigung der materiellen Staatsaufsicht in Europa auf zunehmende Unterstützung getroffen war. ${ }^{22}$ Hier nutze das BMF, unterstützt von der Bundesregierung, also die Tatsache konvergierender Präferenzen im Ministerrat, um eine Regulierungsänderung zu erzielen, für die national die Unterstützung relevanter Akteure fehlte. Der Fall ist ein Beispiel für die von Andrew Moravcsik (1997: 220) vertretene These, dass die Mitgliedschaft in der EU die Regierungen stärkt, weil durch den Wechsel der Verhandlungsarena nationale Vetospieler umgangen werden können.

Mit den dritten Richtlinien kamen große Veränderungen auf das deutsche Versicherungswesen zu, da sich das deutsche Regulierungsmodell hiermit nicht vereinbaren ließ. Parallel zur Durchsetzung der Dienstleistungsfreiheit verboten die dritten Richtlinien die Vorab-Kontrolle von Versicherungsbedingungen und Tarifen, die bis dahin die deutsche Versicherungsregulierung ausmachten. Die Aufsicht bezieht sich jetzt schwerpunktmäßig auf die Solvabilität der Unternehmen. Eine weitere wichtige Veränderung betraf die Monopolversicherer in manchen Bundesländern. Die Monopolanstalten für die Gebäude- und Feuerversicherung, auf die etwa die Hälfte des Prämienvolumens entfiel, mussten aufgelöst werden. Die Bundesländer erwogen anfänglich eine Klage vor dem EuGH, fügten sich dann aber der notwendigen Reform. ${ }^{23}$ Das Beispiel zeigt deutlich, wie durch die europäische Politik das Veto der Bundesländer im Bundesrat umgangen werden kann. Trotz ihrer Reichweite gelang auch die Umsetzung der Reform. Von Seiten des BAV wurde zwar ursprünglich die Kontinuität der Regulierung betont, da sich die Missstandsaufsicht

$22 »$ Versicherungsbedingungen: Russisches Roulett«, Wirtschaftswoche, 21.4.1989; »Keine voreilige Liberalisierung«, Frankfurter Allgemeine Zeitung, 27.4.1989.

23 »Neuordnung der Brandkassen: Private Konkurrenz fürchtet eine Lösung unter einer öffentlich-rechtlichen Ägide«, Handelsblatt, 7.5.1993. 
weiterhin im Gesetz fand. Allerdings sind bereits die Mittel zur Erfüllung der Finanzaufsicht knapp bemessen, so dass darüber hinausgehende Aufgaben kaum bewältigt werden können (int 22). Durch die Abkehr von bisherigen Regulierungsprinzipien änderte sich auch die Rolle der Verbände; ihr Einfluss ging stark zurück, und in der Folge lösten sich die Fachverbände - mit Ausnahme des Verbands der privaten Krankenversicherer - auf und gliederten sich in den GDV ein. ${ }^{24}$ Die Verbände konnten aber die Umsetzung nicht verhindern: Zum einen war ihre Mitarbeit nun kaum noch wichtig, zum anderen entdeckten große Versicherungsgesellschaften zunehmend ihr Interesse am Wettbewerb, womit die Position der Unternehmen uneinheitlicher wurde.

Im Straßengüterverkehr gab es eine Reihe von Anpassungen Anfang der Neunzigerjahre, die schrittweise das deutsche Güterfernverkehrssystem auf den nach dem EuGH-Urteil unabwendbaren Binnenmarkt vorbereiteten. Hervorzuheben ist, dass die Richtlinien selbst diese Schritte nicht verlangten. Sowohl in der bis Ende 1992 gültigen vorläufigen Kabotageregelung als auch in der endgültigen Regelung hatte sich der Ministerrat auf das Tätigkeitslandprinzip geeinigt, so dass die Rechts- und Verwaltungsvorschriften des Aufnahmemitgliedsstaates gelten (Basedow/Held 1990: 307; Gronemeyer 1994: 271). Bei den nationalen Änderungen handelt es sich deshalb um die Reaktion auf einen antizipierten, faktischen Anpassungsdruck: Mit der Einführung der Kabotage wurde die nationale Kontingentregel ebenso wie die Trennung zwischen gewerblichem Güternah- und Güterfernverkehr ausgehöhlt. Man fürchtete, durch eine Beibehaltung der Regelungen deutsche Unternehmer zu benachteiligen, da sie bei ausgeschöpften Kontingenten vom Markt ausgeschlossen worden wären, währenddessen ausländische Unternehmen keine Beschränkung traf (Wissenschaftlicher Beirat 1990: 6; Basedow 1993: 174). Deshalb wurden Fahrzeuge bis 3,5 Tonnen Nutzlast (bisher 0,75 Tonnen) von der Genehmigungspflicht befreit und die Konzessionen für den Güterfernverkehr erheblich ausgeweitet (Aberle 1996: 112). ${ }^{25}$

Eine Koalitionsvereinbarung zu Beginn der Legislaturperiode 1990 sah vor, darüber hinaus die Kontingente und die Tarife ganz aufzuheben. Aber der Bundesverband Deutscher Güterfernverkehr (BDF) sprach sich trotz Kabotage für die Beibehaltung beider Regulierungsprinzipien aus (Zobel 1991: 192f). Auch der zuständige Bundestagsausschuss wandte sich gegen die Aufhebung der Kontingente, so dass dieser Plan aufgegeben wurde. ${ }^{26}$ Hinsichtlich des Tarifsystems lag der Fall anders: Es wurde vom Bundestag zum 1.1.1994 unter dem Eindruck aufgehoben, dass der EuGH in einem Vorlageverfahren des Landgerichts Koblenz die deutschen Tarifkommissionen unter dem europäischen Wettbewerbsrecht für unzulässig erklären

24 Da die private Krankenversicherung noch relativ stark reguliert wird, findet man hier eine Ausnahme.

25 Parallel führte die deutsche Einigung ebenfalls zu einer Ausweitung von Lizenzen (Kerwer/Teutsch 1999: 9).

26 Klemens Weber: »Vom Ausland überrollt? Brummis aus anderen EG-Staaten im Vorteil Fairer Wettbewerb im grenzenlosen Europa noch immer nicht geregelt«, Rheinischer Merkur, 4.12.1992. 
würde (Teutsch 2001: 143f; Héritier 1997: 548). Bei diesem Fall (Rs. 185/91) ging es darum, dass die Reiff GmbH einen Transport zu einem Preis durchführen ließ, der die vorgegebenen Tarife unterschritt. Gleichzeitig verweigerte die Reiff GmbH der BAG die in diesem Fall vorgeschriebene Ausgleichszahlung. Daraufhin ging der Fall vor das Landgericht Koblenz. Hier bemühte sich also ein Verlader, unterstützt vom Bundesverband Werkverkehr und Verlader (BWV), das starre Tarifgefüge aufzubrechen (Teutsch 2001: 143). Da es auch um die Frage ging, ob die deutschen Tarife nach europäischem Recht überhaupt zulässig seien, wurde der Fall dem EuGH vorgelegt. Die Vermutung, dass ein Konflikt zwischen den in Gewerbehand liegenden deutschen Tarifkommissionen und dem Kartellverbot des europäischen Wettbewerbsrechts bestehe, war in der Literatur verbreitet und wurde vom Landgericht Koblenz aufgegriffen (Basedow 1993: 166; Monopolkommission 1990: 307). Es wurde argumentiert, dass die Tarifkommissionen als private Vereinigungen anzusehen seien und unter das europäische Kartellverbot fielen. Die damit bestehende rechtliche Unsicherheit wurde von den Liberalisierungsbefürwortern wie der FDP geschickt genutzt, um eine vorzeitige Änderung des Gesetzes im Bundestag zu erzielen: »Dieser Verflechtung der internationalen Märkte müssen wir durch die Aufhebung der Tarife im Güterverkehr auf nationaler Ebene Rechnung tragen, bevor der EuGH dies qua Urteil macht . $^{27}$

Außer mit der vermeintlichen rechtlichen Notwendigkeit wurde mit dem erwarteten ökonomischen Druck argumentiert. Die Unternehmen müssten auf den kommenden Wettbewerb frühzeitig vorbereitet werden. So passierte die Tarifliberalisierung den Bundestag. Der EuGH seinerseits befand schließlich das deutsche Tarifsystem als rechtmäßig. Unter der Kabotage hätte man das regulierte Tarifsystem sogar ausländischen Unternehmen vorschreiben können. Allerdings wäre die Kontrolle der Tarifeinhaltung bei ausländischen Unternehmen schwierig gewesen, womit die Gefahr einer Inländerdiskriminierung bestand (Teutsch 2001: 142). Tatsächlich hat sich nach Freigabe der Kabotage herausgestellt, dass ihre Nutzung und damit auch der von ihr ausgehende ökonomische Veränderungsdruck - weit hinter den Erwartungen zurückblieben. So machte die Kabotage in der Bundesrepublik weniger als 1\% des kommerziellen Transports aus (Bundesamt für Güterverkehr 1999: 3f). Obwohl rasch deutlich wurde, dass die Reform rechtlich nicht notwendig war, gelang ihre Implementierung. Teutsch (2001: 146) argumentiert, dass sich die Verbände bei der Reform kooperativ verhielten, weil sich die Bundesregierung im Gegenzug auf europäischer Ebene bemüht hatte, die Liberalisierung so lange wie möglich zu verhindern. Zudem erkannte man erst später, dass der von der Kabotage ausgehende Wettbewerbsdruck zu vernachlässigen war.

27 Abgeordneter Horst Friedrich, FDP, Deutscher Bundestag, 12. Wahlperiode, 149. Sitzung, Donnerstag, 25.3.1993, S. 12812A. 


\section{Die nationale Bedingtheit der Folgen der Integration}

Welche Folgen hat die europäische Integration für die Mitgliedsstaaten? Die Verflechtung mit der EU verändert die institutionellen Handlungsoptionen nationaler Akteure, so dass die Folgen der Integration auch national bestimmt sind, ganz wie es die »second-image reversed « Debatte in den Internationalen Beziehungen vermuten lässt (Gourevitch 1978). Wie lässt sich das Ineinandergreifen nationaler und europäischer Faktoren aber genauer fassen, durch das sich die Folgen der Integration bestimmen?

In beiden untersuchten Sektoren war die nationale Regulierung über Jahrzehnte hyperstabil. Damit sind sie beispielhaft für die Reformschwierigkeiten im bundesdeutschen politischen System. Als Ursache ist zunächst der Mesokorporatismus zu nennen, über den politikfeldspezifisch staatliche Steuerung und Selbstorganisation ineinander greifen. Zwar erlaubt die korporatistische Einbindung von Verbänden im Idealfall, Steuerungsziele relativ detailgetreu umzusetzen, jedoch müssen hierfür die Interessen der Verbände auch entsprechend einbezogen werden (Streeck 1987; Scharpf 1988: 76). Sowohl die Versicherungs- als auch die Transportwirtschaft waren aber mit ihren Verbänden gegen eine Reform. Des Weiteren ist das Ressortprinzip wichtig, das Veränderungen schwierig macht, die ihren Ursprung nicht im zuständigen Referat haben. Im Bundesverkehrsministerium fehlte der Wille zu einer weitreichenden Reform; im Bundesfinanzministerium wurde diese erst relativ spät angedacht. Dagegen konnte sich der nur mitbefasste Bundeswirtschaftsminister mit seinen Reformvorstellungen aufgrund der Ressortzuständigkeit nicht durchsetzen. Trotz wachsender Kritik von der Nachfrageseite war in den hier untersuchten Politikfeldern eine Reform gegen die gefestigte Vetokoalition äußerst schwierig. Beim Straßengüterverkehr zählte zu den Reformgegnern auch noch der zuständige Bundestagsausschuss. Zu dem Problem der Vetokoalitionen kam die Zustimmungspflichtigkeit der Gesetze mit der Gefahr, dass sich einige Bundesländer zu Fürsprechern der Reformgegner machen könnten.

$\mathrm{Ab}$ Ende der Achtzigerjahre setzte sich das Bundesfinanzministerium dann für eine Reform der Versicherungsregulierung ein, und mit der Koalitionsvereinbarung von 1991 wurde der Wille der Regierung deutlich, auch die Güterverkehrsregulierung zu reformieren. Durch die Mitgliedschaft in der EU, so das Argument, wurde die Durchsetzung dieser Reformen wesentlich erleichtert. Während der nationale institutionelle Kontext die Akteure der etablierten Vetokoalition begünstigte, wurden im europäischen Mehrebenensystem die Liberalisierungsbefürworter gestärkt. Das Bundesfinanzministerium konnte durch die Unterstützung der dritten Richtlinien auf europäischer Ebene nationale Vetospieler umgehen. Bei der Güterverkehrsliberalisierung wurde die Unsicherheit über das ausstehende EuGHUrteil und die ökonomischen Folgen der Kabotage genutzt. Nun ist der empirische Nachweis schwer zu führen, dass diese Reformen ohne die Mitgliedschaft in der EU so nicht durchsetzbar gewesen wären. National waren die Liberalisierungsgegner institutionell begünstigt, weitreichende Änderungen waren deshalb unwahrscheinlich, wie es eingangs mit Hilfe der analytischen Politik verdeutlicht wurde, denn hier 
hätten entsprechende Zugeständnisse gemacht werden müssen. Während die Versicherungsliberalisierung kaum untersucht ist, kommen Kerwer und Teutsch im StraBengüterverkehr zu dem Fazit: »In the case of Germany, European transport liberalization was in fact vital for overcoming the stability of sectoral corporatism and for the switch to a liberal transport policy approach to be achieved « (Kerwer/Teutsch 2001: 131).

Wie es der EU-Kontext erlaubt, die Vetokoalition zu durchbrechen, lässt sich besonders gut anhand der Güterverkehrsliberalisierung belegen. Aufgrund des äußeren Drucks verlor das federführende Verkehrsministerium sein Monopol über Reforminitiativen. Stattdessen gab es eine Koalitionsvereinbarung. Wahrscheinlich ist, dass die Frage zudem im Kabinett verhandelt wurde. Für beide Foren gilt, dass der Bundesverkehrsminister durch den Übergang der Agendagestaltung sein Vetorecht verlor und sich die Liberalisierungsbefürworter gleichberechtigt einbringen konnten. Im Hinblick auf die Aufhebung der Kontingente scheiterte die Koalitionsvereinbarung im Verkehrsausschuss des Bundestages. Hier konnte sich die alte Vetokoalition also durchsetzen. Anders verlief die Frage der Tarifaufhebung, die unter dem vermeintlichen Druck des EuGH gelang: Die Pro-Liberalisierungsfraktion nutzte das ausstehende Verfahren und die damit veränderte Rückfallposition (vgl. Ostrom 1986: 12f), um ihre Verhandlungsposition zu verbessern; der Status quo der Marktregulierung konnte als nicht mehr stabil dargestellt werden, und eine Reform gelang, die EU-rechtlich, wie sich später herausstellte, gar nicht notwendig gewesen wäre.

Bei der Versicherungsregulierung dagegen kündigte das Fachministerium, unterstützt von der Bundesregierung, die bisherige Koalition auf und setzte sich für die Liberalisierung auf europäischer Ebene ein. Dadurch wurden europäische Vorgaben möglich, für die national der Konsens fehlte, ganz wie es Moravcsik (1997) als Merkmal der Integration behauptet. Die Abschaffung der Versicherungsmonopole der Länder ist hierfür ein besonders deutliches Beispiel - im Bundesrat wäre diesbezüglich die Zustimmung verweigert worden. Dasselbe lässt sich aber auch für die sehr weitgehende Liberalisierung des Aufsichtsrechts vermuten, gegen die sich die Versicherungswirtschaft gemeinsam mit dem Bundesaufsichtsamt vehement zur Wehr setzte.

Wie aber lassen sich solche weitgehenden Reformen national implementieren? Sind es eventuell nur bestimmte Reformschritte, die gegen den Willen wichtiger Akteure so veranlasst werden können? Bevor diese Fragen diskutiert werden, soll zunächst nach den Verallgemeinerungsmöglichkeiten gefragt werden. Die Verflechtung mit der EU kann von nationalen Akteuren in all den Fällen zur Stärkung ihrer Verhandlungsposition benutzt werden, wo durch europäische Politik das nationale Kräftegleichgewicht verschoben wird. Das kann zunächst einmal, wie das Beispiel der Versicherungen zeigt, in den verschiedensten Politikbereichen der Fall sein, wenn das federführende Ministerium, gestützt von der Bundesregierung, im Ministerrat Änderungen unterstützt, die national höchst wahrscheinlich blockiert worden wären. Neben dieser vergleichsweise wirksamen Einflussnahme über den Ministerrat, die aber nur der Regierung offen steht, können andere nationale Akteure versu- 
chen, über die Kommission, das Europäische Parlament oder den Ministerrat Entscheidungen zu beeinflussen. Alleine können sie aber weitreichende Veränderungen so kaum bewirken. Darüber hinaus können nationale Akteure in den Fällen das europäische System benutzen, in denen es relativ eindeutig bestimmte Rechte und Pflichten formuliert, so dass der EuGH angerufen werden kann. Dies ist vor allem im Bereich der so genannten negativen Integration der Fall, da die Pflicht der Mitgliedsstaaten zur Herstellung der vier Freiheiten (von Waren, Dienstleistungen, Arbeit und Kapital) und zur Durchsetzung der Wettbewerbsordnung in den Verträgen niedergelegt ist (Scharpf 1999: 52-69). Hier kann europäisches Richterrecht greifen, das Akteuren die Möglichkeit bietet, gegen unliebsame nationale Regulierungen gerichtlich vorzugehen. In den hier behandelten beiden Fällen folgte die gerichtliche Auseinandersetzung um das deutsche Güterverkehrstarifsystem beim Landgericht Koblenz diesem Muster. ${ }^{28}$ Aufgrund der einseitigen Ausrichtung des Europäischen Vertragswerks kann dieses eher dazu genutzt werden, nationale Regulierungen zu Fall zu bringen als zusätzliche Regulierungen einzufordern. ${ }^{29}$ Insofern werden nur bestimmte Akteursinteressen gefördert. Auch in anderen als den hier behandelten Politikfeldern konnten so bestehende Reformblockaden überwunden werden. Besonders deutlich lässt sich dies in der Telekommunikationspolitik beobachten, in der Deutschland zunächst wegen der Vetoposition des Bundesrates Mühe hatte, das Monopol aufzugeben (Schmidt 1991). Im Verlauf der weiteren Reform nutzten Teile des zuständigen Postministeriums beispielsweise die Verbindungen zur Kommission, um die Liberalisierung des Sprachtelefondienstes voranzutreiben. Auf die deutsche Bitte hin erfolgte ein Brief aus Brüssel, der mit seiner Interpretation der Rechtslage die Einigung auf eine liberalere Regelung national erleichterte (Schmidt 1998: 151f). Akteure können die Einbindung ins europäische Mehrebenensystem so nutzen, um ihre Durchsetzungsfähigkeit gegenüber nationalen Vetospielern zu verbessern.

Auch von Knill und Lehmkuhl (1999) wird die für nationale Akteure veränderte Opportunitätsstruktur in Fällen negativer Integration ${ }^{30}$ hervorgehoben. Darüber hinaus argumentieren sie, bei negativer Integration solle auf Akteursstrategien und nur bei positiver Integration auf die institutionelle Passgenauigkeit abgestellt werden. Denn in Fällen negativer Integration handele es sich um nur sehr lose europäische

28 Nationale Akteure können sich entweder direkt bei der Kommission über Verletzungen des europäischen Wettbewerbsrechts beschweren oder sie klagen vor deutschen Gerichten, die im Zweifelsfall die Frage dem EuGH vorlegen (Schmidt 1998: 72). Ein weiteres aktuelles Beispiel hierfür ist das Zufallbringen der Gewährträgerhaftung der öffentlichen Banken durch die privaten Banken in Deutschland, indem sich die privaten Banken bei der Europäischen Kommission über die daraus folgenden Wettbewerbsnachteile beschwerten.

29 Eine Ausnahme ist hier der Artikel 141 (ex 119) des Vertrags, der vielfältig zur Verbesserung der Situation von Frauen genutzt wurde (Tesoka 1999).

30 Die Unterscheidung nach negativer und positiver Integration ist problematisch wegen ihrer doppelten Stoßrichtung. Zum einen geht es um den Inhalt der Regel (marktschaffend versus marktgestaltend), zum anderen um den Prozess des Zustandekommens (EuGH versus Ministerrat). Schließlich gehen negative und positive Integration Hand in Hand, so dass ganze Politikfelder kaum in diese Kategorien einzuordnen sind. 
Vorgaben, so dass es darauf ankomme, wie Akteure diese nutzten. Bei Maßnahmen positiver Integration seien die europäischen Vorgaben im Gegenteil relativ fest definiert. Die Europäisierungswirkungen bestimmten sich deshalb primär aus der institutionellen Kompatibilität zwischen bestehenden nationalen Regeln und neuen europäischen Vorgaben. Dieser Vorschlag scheint durch die Fallauswahl mitbeeinflusst: Ihr Beispiel für negative Integration, die auch hier diskutierte Straßengüterverkehrsliberalisierung, kann als ein Beispiel für eine sehr autonomieschonende Integration angesehen werden (Schmidt 2002), weil hier der Ministerrat das Tätigkeitslandprinzip für die Kabotage vorschrieb und auch der EuGH die nationale Tarifsetzung nicht antastete. Typischer ist dagegen, dass Vorgaben negativer Integration auf nationaler Ebene kaum Anpassungsspielraum lassen: Bestimmte staatliche Maßnahmen in Form von Regulierungen oder Beihilfen werden unter Hinweis auf höher stehendes europäisches Recht unterbunden. Anders als Knill und Lehmkuhl (1999: 2, 8) argumentieren, handelt es sich hier nicht um beschränkte institutionelle Auswirkungen, da schließlich über negative Integration nationale Monopole von öffentlichen Dienstleistungen ebenso gebrochen werden können wie Schwerpunkte nationaler Wirtschaftsförderung oder selbst die staatlich regulierten Sozialsysteme (Ehlermann 1993; Leibfried/Pierson 2000). Dagegen erscheint die Anpassung an Maßnahmen positiver Integration insofern leichter, als hier die Regierungen im Ministerrat an der Entscheidung immerhin beteiligt sind und gegebenenfalls durch die Einfügung verschiedener Optionen darauf hinwirken können, dass keine allzu großen nationalen Veränderungen verlangt werden. So ist allgemein anerkannt, dass trotz Mehrheitsabstimmungen im Ministerrat Mitgliedsstaaten bei vitalen nationalen Interessen nicht überstimmt werden (Westlake 1995: 111).

Angesichts dessen ist es plausibel, dass es für die Auswirkungen sowohl der marktschaffenden als auch der marktgestaltenden (also negativer und positiver) Integration auf die institutionell geforderten Anpassungsleistungen und die Interessen der beteiligten Akteure ankommt. Gerade das Beispiel des Straßengüterverkehrs zeigt, dass national Anpassungsdruck zum einen (institutionell) aufgrund eindeutig definierter europäischer Vorgaben erfolgt und zum anderen aufgrund der von Akteuren antizipierten Folgen der europäischen Integration (in diesem Falle die Rechtsprechung des EuGH und der Wettbewerbsdruck durch die Kabotage). Das Beispiel der Versicherung macht deutlich, dass zudem Regierungsvertreter (auf der Basis konvergierender Präferenzen mit den anderen Mitgliedsstaaten) im Ministerrat nationale Vetopunkte umgehen können.

Damit scheint eine Unterscheidung sinnvoll, die wie bei Knill und Lehmkuhl (1999) zum einen auf den institutionellen Anpassungsdruck und zum anderen auf Akteursstrategien abstellt, ohne jedoch gleichzeitig nach positiver und negativer Integration zu differenzieren. Angelehnt an Radaelli (2001: 124) kann man eine vertikale Dimension von Europäisierung ausmachen, bei der es sich um eindeutige europäische Vorgaben handelt, denen auf nationaler Ebene entsprochen werden muss. In der horizontalen Dimension dagegen kommen Akteursstrategien stärker zum Tragen. Hier bietet die Überlagerung der nationalen mit der europäischen Arena nationalen Akteuren eine veränderte Opportunitätsstruktur, die sie nutzen 
können, um Interessen durchzusetzen, die auf nationaler Ebene nicht durchsetzbar wären. Dabei handelt es sich nicht um klar umrissene europäische Vorgaben wie bei der vertikalen Dimension, sondern um die Reaktion auf potenzielle ökonomische oder rechtliche Folgen von Integrationsentscheidungen oder um Möglichkeiten politischer Einflussnahme, z. B. über den Ministerrat. Nationale Akteure können hier einen sich aus der Integration ergebenden Handlungsspielraum strategisch nutzen. Dies betrifft die Antizipation und Deutung möglicher ökonomischer Folgen des Wettbewerbs ebenso wie eventuelle Urteile des EuGH und die Möglichkeit, politisch auf die europäischen Organe Einfluss zu nehmen. Es leuchtet ein, dass sich nur Aspekte europäischer Policies in die Dimensionen einordnen lassen, und nicht ganze Sektorpolitiken (wie auch bereits für die Einordnung nach positiver und negativer Integration argumentiert wurde).

Hierzu passt, dass es bei beiden Fällen national zu einer Überschreitung der Brüsseler Vorgaben kam. Auch hieran wird deutlich, dass sich die Politikprozesse durch das Ineinandergreifen europäischer und nationaler Faktoren erklären, und nicht durch einseitige Anpassung. So ging man bei der Implementation der zweiten Schadensrichtlinie $^{31}$ über die Vorgaben der Richtlinie hinaus. Anders als beim Straßengüterverkehr, als die Unsicherheit über das ausstehende EuGH-Urteil geschickt ausgenutzt wurde, waren in diesem Fall die europarechtlichen Vorgaben aber unzweifelhaft. Auch in anderen Bereichen wurden in der Bundesrepublik im Zuge des durch die EU notwendigen Wandels sehr weitgehende Änderungen durchgesetzt, zum Beispiel in der Telekommunikation sowie der Elektrizitäts- und Bahnliberalisierung (Eberlein/Grande 2000: 46f) ${ }^{32}$ Da es unwahrscheinlich ist, dass gerade im Moment der europäischen Reform die relevanten nationalen Akteure eine weitgehende Liberalisierung präferieren, ist zu vermuten, dass auch diese Fälle dem Muster der hier vorgestellten Beispiele folgen. Durch die vielfältigen institutionellen Vetopunkte in der Bundesrepublik sind einmal vereinbarte Sektorregulierungen äußerst stabil, selbst wenn sie nicht mehr dem Gleichgewicht der relevanten Akteursinteressen entsprechen. Policies können auch von einer Minderheit von Akteuren getragen werden; die Verflechtung mit der EU erlaubt es den national unterlegenen Akteuren, ihre Interessen in einer anderen Arena zu verfolgen. Da auf diese Weise faktische und institutionalisierte Vetopunkte umgangen werden können (regulierte Unternehmen und ihre Verbände, Bundesaufsichtsamt sowie evtl. das Fachministerium und die Bundesländer), kann es zu einer sehr weitgehenden Änderung kommen. Diese zeigt, wie wenig der Status quo noch die Interessen relevanter Akteure reflektierte. Nach dem Verlust der Vetomöglichkeiten konnten die Status-

31 Bei den 3. Richtlinien ist das Bild weniger eindeutig: Einerseits wurden manche Mindeststandards überschritten, andererseits unterblieb aber die Regulierung der Versicherungsvermittlung, die als ein notwendiges Komplement zur Aufgabe der Bedingungsgenehmigung angesehen werden kann.

32 Da die Elektrizitätsnetze in Deutschland über Sektorvereinbarungen geöffnet werden, ist teils umstritten, wie liberal das System tatsächlich ist. Ungeachtet dieser Schwierigkeiten hat die deutsche Reform selbst privaten Haushalten von Anfang an den Produzentenwechsel erlaubt, womit es sich ohne Zweifel um einen radikalen Schnitt handelt. 
quo-Befürworter kaum noch auf das Ergebnis Einfluss nehmen. Im Gegenzug wurde erst mit der Umgehung verschiedener Vetopunkte das Gewicht der Liberalisierungsbefürworter deutlich.

Die Diskussion über Verallgemeinerungsmöglichkeiten zeigt, dass zwar nur bestimmte Reformen durch die Verknüpfung der nationalen mit der europäischen Arena erleichtert werden, diese aber durchaus weitreichende Verteilungswirkungen haben können. Zwar handelt es sich bei Regulierungsänderungen im Prinzip um Koordinationsspiele, aber durch eine Änderung verlieren manche Akteure erhebliche Privilegien. Wie erklärt es sich, dass die Implementierung national dennoch gelingt? Die quasi-hierarchische Kontrolle des EuGH ist in den hier diskutierten Fällen ja insofern unzureichend, als es sich nicht um rein europäische erzwungene Änderungen handelt. Zum einen - dies zeigt der Güterverkehr - gelingt die Umsetzung (abgesehen von der zunächst vermuteten rechtlichen Notwendigkeit) in Erwartung eines ökonomischen Drucks, der falsch eingeschätzt wurde. Nur mit falschen Erwartungen sind aber die Reformen ähnlichen Musters in den verschiedenen Sektoren nicht zu erklären. $\mathrm{Zu}$ der teils vermuteten, teils vorgespiegelten, durch Europa bedingten Veränderungsnotwendigkeit kommt zum anderen, dass zentrale Akteure ihre Interessen neu bewerten. So erkannten die großen Versicherer wie die Allianz, dass die Liberalisierung ihnen Wachstumschancen auf Kosten der kleinen Versicherer bot. Aus ähnlichen Gründen kündigten die großen Energieversorger die Koalition mit den kleinen Unternehmen (Eising 2002: 100). ${ }^{33}$ In der Telekommunikation erkannten zunächst die Gerätehersteller, dass ihnen die Liberalisierung mit den neuen Wettbewerbern neue Kunden versprach. Aber auch die Telekom sah, dass der Verlust von Privilegien wie des Versorgungsmonopols die Lockerung von Versorgungsverpflichtungen erlaubte und zudem neue Expansionsmöglichkeiten im Ausland beinhaltete. Hinzu kommt, dass die Liberalisierung gerade für die Unternehmenseliten Vorteile bietet: Die Interessenneubewertung großer Unternehmen im Zuge der Reform erklärt, weshalb die Implementierung gelingen konnte, obwohl mit den Verbänden zentrale Akteure an Einfluss verloren. Insofern spielt bei der Umsetzung auch Lernen eine Rolle.

Ergibt sich aus dem nationalen Institutionengefüge eine Tendenz zum Reformstau wie in Deutschland, können die mit Europa verknüpften Veränderungen also entsprechend weitreichend sein. Die vermehrten Handlungsoptionen innerhalb des europäischen Mehrebenensystems erlauben es, nationale Reformblockaden aufzubrechen. Es kommt zu Wandel durch Verflechtung. Für die Analyse der Folgen der Integration zeigt dieser Mechanismus anschaulich, dass sich diese nicht nur aus der Frage von Inkompatibilitäten und Anpassungsdruck bestimmen. Vielmehr erzeugt die Mitgliedschaft in der EU auf nationaler Ebene Interaktionseffekte, die mit dem Begriff der Resonanz umschrieben werden können. Nur so erklärt sich, welche

33 Eising (2002) analysiert die nationale Überimplementation von EU-Vorgaben anhand der europäischen Elektrizitätsliberalisierung als Ausdruck EU-induzierten Lernens. Dagegen vernachlässigt er die Frage, inwieweit der Status quo ante angesichts vielfältiger Vetopunkte überhaupt noch die relevanten Akteursinteressen widerspiegelte. 
Handlungsoptionen national aufgegriffen werden und wie die Mehrebenenregulierung national gestaltet wird. Insofern sind die Folgen der Integration in nicht geringem Maße auch national bestimmt. Für die Konzeptualisierung des europäischen Mehrebenensystems bedeutet dies, dass das Ineinandergreifen verschiedener institutioneller Ebenen nicht linear gedacht werden kann. Abhängig von den nationalen institutionellen Bedingungen ergeben sich Interaktionseffekte zwischen nationaler und europäischer Ebene, die für die konkreten Folgen der Integration bestimmend werden.

\section{Literatur}

Aberle, Gerd 1987: Sektorale Deregulierung. Die EG-Integration erzwingt veränderte ordnungspolitische Strukturen auf den Straßengüterverkehrsmärkten, in: Hamburger Jahrbuch für Wirtschaft- und Gesellschaftspolitik 32, Hamburg, 157-174.

Aberle, Gerd 1996: Transportwirtschaft: einzelwirtschaftliche und gesamtwirtschaftliche Grundlagen, München.

Badenhoop, Jörn H.-E. 1988: Binnenmarkt der Versicherungen, in: Weidenfeld, Werner (Hrsg.): Binnenmarkt '92: Perspektiven aus deutscher Sicht, Gütersloh, 103-106.

Basedow, Jürgen 1993: EG-Recht und Verkehrspolitische Handlungsspielräume der Mitgliedsstaaten nach 1992, in: Herber, Rolf (Hrsg.): Das deutsche Transportrecht an der Schwelle zum Europäischen Binnenmarkt (Symposium der Deutschen Gesellschaft für Transportrecht, Wiesbaden 1991), Neuwied, 155-179.

Basedow, Jürgen/Dolfen, Michael 1998: Verkehrs- und Transportrecht, in: Dauses, Manfred A. (Hrsg.): Handbuch des EU-Wirtschaftsrechts, München, 1-148.

Basedow, Jürgen/Held, Ansgar 1990: Die EG-Kabotageverordnung. Ein Schritt zur Verwirklichung der Dienstleistungsfreiheit im Straßengüterverkehr, in: Europäische Zeitschrift für Wirtschaftsrecht 5, 305-308.

Börzel, Tanja A. 2002: Pace-Setting, Foot-Dragging, and Fence-Sitting: Member State Responses to Europeanization, in: Journal of Common Market Studies 40: 2, 193-214.

Börzel, Tanja A./Risse, Thomas 2000: When Europe hits Home: Europeanization and Domestic Change, in: European Integration online Papers 4: 15, http://eiop.or.at/eiop/texte/ 2000-015a.htm; 17.03.2003.

Bujard, Helmut 1991: Zu den Konsequenzen des Paradigmawechsels der Versicherungspolitik, in: Zeitschrift für das Versicherungswesen 41: 8, 191-194.

Bundesamt für Güterverkehr 1999: Marktbeobachtung Güterverkehr. Sonderbericht: Die Auswirkungen der weiteren Liberalisierung des europäischen Verkehrsmarktes im Jahr 1998 auf die Unternehmen des gewerblichen Güterkraftverkehrs, in: http://www.bag.bund.de/ service/Liberalisierung.pdf; 17.03.2003.

Button, Kenneth J. 1984: Road Haulage Licensing and EC Transport Policy, Hamshire.

Czada, Roland 2000: Dimensionen der Verhandlungsdemokratie, (Arbeitspapiere des Instituts für Politikwissenschaft der Fernuniversität Hagen, Band 46), Hagen.

Deregulierungskommission 1991: Marktöffnung und Wettbewerb, Stuttgart.

Dimitrova, Antoaneta/Steunenberg, Bernard 2000: The Search for Convergence of National Policies in the European Union: An Impossible Quest?, in: European Union Politics 1: 2, 201-226.

Dreher, Meinrad 1996: Die Quersubventionierung bei Versicherungsunternehmen nach europäischem und deutschem Versicherungsrecht, in: Zeitschrift für die gesamte Versicherungswissenschaft 85, 499-520. 
Eberlein, Burkard/Grande, Edgar 2000: Regulation and Infrastructure Management: German Regulatory Regimes and the EU Framework, in: German Policy Studies/Politikfeldanalyse 1: 1, 39-66.

Eggerstedt, Harald 1986: Deregulierung der Versicherungswirtschaft. Ein grundlegender Reformansatz (Aufsätze zur Wirtschaftspolitik Nr. 15), Mainz.

Ehlermann, Claus-Dieter 1993: Wettbewerbspolitik im Binnenmarkt, in: Recht der Internationalen Wirtschaft 39: 10, 793-797.

Eising, Rainer 2002: Policy Learning in Embedded Negotiations: Explaining EU Electricity Liberalization, in: International Organization 56: 1, 85-120.

Erdmenger, Jürgen 1985: Die EG-Verkehrspolitik vor Gericht. Das EuGH-Urteil Rs. 13/83 vom 22.5.1985 und seine Folgen, in: Europarecht 20: 4, 375-392.

Everson, Michelle 1996: The German Federal Supervisory Authority for Insurance, in: Majone, Giandomenico (Hrsg.): Regulating Europe, London, 202-228.

Feick, Jürgen/Klaes, Lothar/Lex, Christa/Mayntz, Renate/Seebach, Rolf 1982: Regulative Politik und politisch-administrative Kultur. Ein Vergleich von fünf Ländern und vier Interventionsprogrammen, Köln.

Finanzausschuss 1990: Öffentliche Anhörung zum Entwurf eines Gesetzes zur Durchführung versicherungsrechtlicher Richtlinien des Rates der Europäischen Gemeinschaften (zweites Durchführungsgesetz/EWG zum VAG), in: Protokoll der öffentlichen Sitzung des Finanzausschusses.

Garlichs, Dietrich/Müller, Edda 1977: Eine neue Organisation für das Bundesverkehrsministerium, in: Die Verwaltung 10: 3, 343-362.

Gärtner, Rudolf 1984: Versicherungen, in: Oberender, Peter (Hrsg.): Marktstruktur und Wettbewerb in der Bundesrepublik Deutschland. Branchenstudien zur deutschen Volkswirtschaft, München, 491-535.

Geiger, Hermann 1992: Der Schutz der Versicherten im Europäischen Binnenmarkt, Konstanz.

Gourevitch, Peter 1978: The Second Image Reversed: The International Sources of Domestic Politics, in: International Organization 32: 4, 881-911.

Gronemeyer, Nils 1994: Die Entwicklung des EU-Kabotage-Rechts bis zur neuen KabotageVerordnung (EWG) Nr. 3118/93, in: Transportrecht 17, 267-271.

Hamm, Walter 1984: Transportwesen, in: Oberender, Peter (Hrsg.): Marktstruktur und Wettbewerb in der Bundesrepublik Deutschland. Branchenstudien zur deutschen Volkswirtschaft, München, 455-489.

Hamm, Walter 1992: Durchbruch in der Verkehrsderegulierung, in: Hamburger Jahrbuch für Wirtschafts- und Gesellschaftspolitik 37, Hamburg, 225-243.

Héritier, Adrienne 1997: Market-making Policy in Europe: Its Impact on Member State Policies. The Case of Road Haulage in Britain, the Netherlands, Germany and Italy, in: Journal of European Public Policy 4: 4, 539-555.

Hinich, Melvin J./Munger, Michael C. 1997: Analytical Politics, Cambridge.

Hollenders, Christoph 1985: Die Bereichsausnahme für Versicherungen nach § 102 GWB, Baden-Baden.

Katzenstein, Peter J. 1987: Policy and Politics in West Germany, Philadelphia, PA.

Kerwer, Dieter/Teutsch, Michael 1999: Coping with Integration: The Europeanization of Road Haulage Policy in France, Germany, and Italy, unv. Ms.

Kerwer, Dieter/Teutsch, Michael 2001: Elusive Europeanization: Liberalizing Road Haulage in the European Union, in: Journal of European Public Policy 8: 1, 124-143.

Knill, Christoph/Lehmkuhl, Dirk 1999: How Europe Matters: Different Mechanisms of Europeanization, in: European Integration online Papers 3, http://eiop.or.at/eiop/texte/1998007a.htm; 17.03.2003. 
Knill, Christoph/Lenschow, Andrea 1998: Coping with Europe: The Impact of British and German Administrations on the Implementation of EU Environmental Policy, in: Journal of European Public Policy 5: 4, 595-614.

Knudsen, Jette Steen 2001: Breaking with Tradition: Liberalization of Service Trade in the European Union, Cambridge, MA.

Krakowski, Michael 1988: Regulierung der Versicherungsmärkte, in: Krakowski, Michael (Hrsg.): Regulierung in der Bundesrepublik Deutschland. Die Ausnahmebereiche des Gesetzes gegen Wettbewerbsbeschränkungen, Hamburg, 423-497.

Laaser, Claus-Friedrich 1991: Wettbewerb im Verkehrswesen. Chancen für eine Deregulierung in der Bundesrepublik, Tübingen.

Lehmkuhl, Dirk 1998: The Importance of Small Differences: The Impact of European Integration on the Associations in the German and Dutch Road Haulage Industries, Florenz.

Leibfried, Stephan/Pierson, Paul 2000: Social Policy, in: Wallace, Helen/Wallace, William (Hrsg.): Policy-making in the European Union, Oxford, 267-292.

Luthardt, Wolfgang 1999: Abschied vom deutschen Konsensmodell? Zur Reform des Föderalismus, in: Aus Politik und Zeitgeschichte 49: 13, 12-23.

Mayntz, Renate/Scharpf, Fritz W. 1975: Policy Making in the German Federal Bureaucracy, Amsterdam.

Moe, Terry M./Caldwell, Michael 1994: The Institutional Foundations of Democratic Government: A Comparison of Presidential and Parliamentary Systems, in: Journal of Institutional and Theoretical Economics 150: 1, 171-195.

Monopolkommission 1988: Die Wettbewerbsordnung erweitern. Hauptgutachten 1986/87, Baden-Baden.

Monopolkommission 1990: Wettbewerbspolitik vor neuen Herausforderungen. Hauptgutachten 1988/89, Baden-Baden.

Moravcsik, Andrew 1997: Warum die Europäische Union die Exekutive stärkt: Innenpolitik und internationale Kooperation, in: Wolf, Klaus Dieter (Hrsg.): Projekt Europa im Übergang?, Baden-Baden, 211-269.

Müller, Jürgen/Vogelsang, Ingo 1979: Staatliche Regulierung. Regulated Industries in den USA und Gemeinwohlbindung in wettbewerblichen Ausnahmebereichen in der Bundesrepublik Deutschland, Baden-Baden.

Müller-Rommel, Ferdinand 1988: Interessengruppenvertretung im Deutschen Bundestag, in: Thaysen, Uwe/Davidson, Roger H./Livingston, Robert G. (Hrsg.): US-Kongreß und Deutscher Bundestag, Opladen, 300-323.

Ostrom, Elinor 1986: An Agenda for the Study of Institutions, in: Public Choice 48: 1, 3-25.

Peltzman, Sam 1989: The Economic Theory of Regulation after a Decade of Deregulation, in: Brookings Papers on Economic Activity 1, 1-41.

Przeworski, Adam/Teune, Henry 1985: The Logic of Comparative Social Inquiry, Malabar.

Radaelli, Claudio M. 2001: The Domestic Impact of European Union Public Policy: Notes on Concepts, Methods, and the Challenge of Empirical Research, in: Politique européenne $5,107-142$.

Scharpf, Fritz W. 1985: Die Politikverflechtungsfalle. Europäische Integration und Deutscher Föderalismus im Vergleich, in: Politische Vierteljahresschrift 26: 4, 323-356.

Scharpf, Fritz W. 1988: Verhandlungssysteme, Verteilungskonflikte und Pathologien der politischen Steuerung, in: Schmidt, Manfred G. (Hrsg.): Staatstätigkeit: International und historisch vergleichende Analysen (PVS-Sonderheft 19), Opladen, 61-87.

Scharpf, Fritz W. 1989: Der Bundesrat und die Kooperation auf der »dritten Ebene«, in: Bundesrat (Hrsg.): Vierzig Jahre Bundesrat, Baden-Baden, 121-166.

Scharpf, Fritz W. 1999: Regieren in Europa. Effektiv und demokratisch?, Frankfurt a. M. 
Schmidt, Susanne K. 1991: Taking the Long Road to Liberalization. Telecommunications Reform in the Federal Republic of Germany, in: Telecommunications Policy 15: 3, 209222.

Schmidt, Susanne K. 1998: Liberalisierung in Europa. Die Rolle der Europäischen Kommission, Frankfurt a. M.

Schmidt, Susanne K. 2000: Only an Agenda Setter? The European Commission's Power over the Council of Ministers, in: European Union Politics 1: 1, 37-61.

Schmidt, Susanne K. 2002: The Impact of Mutual Recognition - Inbuilt Limits and Domestic Responses to the Single Market, in: Journal of European Public Policy 9: 6, 935-953.

Schmidt, Vivien A. 1997: European Integration and Institutional Change: The Transformation of National Patterns of Policy-making, in: Göhler, Gerhard (Hrsg.): Institutionenwandel, Opladen, 143-180.

Schmidt, Vivien A. 1999: The EU and Its Member-States: Institutional Contrasts and Their Consequences (MPIfG Working Paper 99/7), Köln.

Schmitt, Veit 1993: Die Harmonisierung der Wettbewerbsbedingungen in der EG-Binnenverkehrspolitik. Eine Bilanz der technischen, sozialen und steuerlichen Rechtsetzung, in: Europäische Zeitschrift für Wirtschaftsrecht 8, 305-311.

Starke, Otto-Ernst 1988: Umfassende Deregulierung in Versicherungswirtschaft und Versicherungsaufsicht gefordert, in: Versicherungswirtschaft 43: 18, 1226-1239.

Stigler, George 1971: The Theory of Economic Regulation, in: Bell Journal of Economics and Management Science 2: 1, 3-21.

Streeck, Wolfgang 1987: Vielfalt und Interdependenz. Überlegungen zur Rolle von intermediären Organisationen in sich ändernden Umwelten, in: Kölner Zeitschrift für Soziologie und Sozialpsychologie 39: 3, 471-495.

Tesoka, Sabrina 1999: Judicial Politics in the European Union: Its Impact on National Opportunity Structures for Gender Equality (MPIfG Discussion Paper 99/2), Köln.

Teutsch, Michael 2001: Regulatory Reforms in the German Transport Sector: How to Overcome Multiple Veto Points, in: Héritier, Adrienne/Kerwer, Dieter/Knill, Christoph/ Lehmkuhl, Dirk/Teutsch, Michael/Douillet, Anne-Cécile (Hrsg.): Differential Europe, Lanham, MD, 133-172.

Wachendorfer-Schmidt, Ute 1999: Der Preis des Föderalismus in Deutschland, in: Politische Vierteljahresschrift 40: 1, 3-39.

Wacker-Theodorakopoulos, Cora 1988: Regulierung des Verkehrssektors, in: Krakowski, Michael (Hrsg.): Regulierung in der Bundesrepublik Deutschland. Die Ausnahmebereiche des Gesetzes gegen Wettbewerbsbeschränkungen, Hamburg, 287-345.

Westlake, Martin 1995: The Council of the European Union, London.

Willeke, Rainer 1997: Verkehrswissenschaft als Begleiter der Verkehrsentwicklung und Verkehrspolitik, in: Zeitschrift für Verkehrswissenschaft 68: 1, 52-72.

Wissenschaftlicher Beirat 1990: Kabotage im Straßengüterverkehr. Gutachten des Wissenschaftlichen Beirates beim Bundesminister für Verkehr - Gruppe Verkehrswirtschaft, in: Zeitschrift für Verkehrswissenschaft 61: 1, 3-14.

Zobel, Adolf 1991: Droht im Güterkraftverkehr ein Ordnungsdualismus? in: DVG (Hrsg.): Internationaler Verkehr. Europa am Ende des Jahrtausends, Bergisch-Gladbach, 178193. 